\title{
Poor sleep quality among newly diagnosed head and neck cancer patients: prevalence and associated factors
}

\author{
Angelina M. M. Santoso ${ }^{1,2}$ (1) - Femke Jansen ${ }^{1,2,3} \cdot$ Birgit I. Lissenberg-Witte ${ }^{4}$. Robert J. Baatenburg de Jong ${ }^{5}$. \\ Johannes A. Langendijk ${ }^{6}$. C. René Leemans ${ }^{3}$. Johannes H. Smit ${ }^{7} \cdot$ Robert P. Takes $^{8} \cdot$ Chris H. J. Terhaard $^{9}$. \\ Annemieke van Straten ${ }^{1} \cdot$ Irma M. Verdonck-de Leeuw ${ }^{1,2,3} \cdot$ the NET-QUBIC consortium $^{10}$
}

Received: 26 February 2020 / Accepted: 11 June 2020 / Published online: 22 June 2020

(C) The Author(s) 2020

\begin{abstract}
Background Head and neck cancer (HNC) patients often suffer from distress attributed to their cancer diagnosis which may disturb their sleep. However, there is lack of research about poor sleep quality among newly diagnosed HNC patients. Therefore, our aim was to investigate the prevalence and the associated factors of poor sleep quality among HNC patients before starting treatment.

Materials and methods A cross-sectional study was conducted using the baseline data from NET-QUBIC study, an ongoing multicenter cohort of HNC patients in the Netherlands. Poor sleep quality was defined as a Pittsburgh Sleep Quality Index (PSQI) total score of $>5$. Risk factors examined were sociodemographic factors (age, sex, education level, living situation), clinical characteristics (HNC subsite, tumor stage, comorbidity, performance status), lifestyle factors, coping styles, and HNC symptoms.

Results Among $560 \mathrm{HNC}$ patients, 246 (44\%) had poor sleep quality before start of treatment. Several factors were found to be significantly associated with poor sleep: younger age (odds ratio [OR] for each additional year 0.98, 95\% CI 0.96-1.00), being female (OR 2.6, 95\% CI 1.7-4.1), higher passive coping style (OR 1.18, 95\% CI 1.09-1.28), more oral pain (OR 1.10, 95\% CI 1.01-1.19), and less sexual interest and enjoyment (OR 1.13, 95\% CI 1.06-1.20).

Conclusion Poor sleep quality is highly prevalent among HNC patients before start of treatment. Early evaluation and tailored intervention to improve sleep quality are necessary to prepare these patients for HNC treatment and its consequences.
\end{abstract}

Keywords Sleep quality $\cdot$ Head and neck cancer $\cdot$ Newly diagnosed $\cdot$ Before treatment

\section{Abbreviations}

ACE-27

BMI
Adult comorbidity evaluation

Body mass index
ECOG

Irma M. Verdonck-de Leeuw

im.verdonck@amsterdamumc.nl

1 Department of Clinical, Neuro- and Developmental Psychology, Faculty of Behavioural and Movement Sciences \& Amsterdam Public Health Research Institute, Vrije Universiteit Amsterdam, Van der Boechorststraat 7, 1081 BT Amsterdam, The Netherlands

2 Cancer Center Amsterdam Research Institute, Amsterdam UMC, Vrije Universiteit Amsterdam, Amsterdam, The Netherlands

3 Department of Otolaryngology - Head and Neck Surgery, Amsterdam UMC, Vrije Universiteit Amsterdam, PO Box 7057, 1007 MB Amsterdam, The Netherlands

4 Department of Epidemiology and Biostatistics, Amsterdam UMC, Vrije Universiteit Amsterdam, Amsterdam, The Netherlands
Eastern cooperative oncology group
5 Department of Otolaryngology and Head and Neck Surgery, Erasmus Cancer Institute, Erasmus MC, Rotterdam, The Netherlands

6 Department of Radiation Oncology, University of Groningen, University Medical Centre Groningen, Groningen, The Netherlands

7 Department of Psychiatry, Amsterdam Public Health, Amsterdam UMC, Vrije Universiteit Amsterdam, Amsterdam, The Netherlands

8 Department of Otorhinolaryngology and Head and Neck Surgery, Radboud Institute for Health Sciences, Radboud University Medical Center, Nijmegen, The Netherlands

9 Department of Radiotherapy, University Medical Center, Utrecht, The Netherlands

10 Department of Otolaryngology - Head and Neck Surgery, Project Kubus, Amsterdam UMC, Vrije Universiteit Amsterdam, Amsterdam, The Netherlands 
EORTC QLQ-H\&N35 European organization for research and treatment of cancer quality of life questionnaire - HNC-specific module

$\begin{array}{ll}\text { HNC } & \text { Head and neck cancer } \\ \text { HRQoL } & \begin{array}{l}\text { Health-related quality of life } \\ \text { Interquartile range } \\ \text { IQR }\end{array} \\ \text { The NETherlands QUality of life } \\ \text { and Blomedical Cohort study in } \\ \text { head and neck cancer } \\ \text { Physical activity scale for the } \\ \text { PASE } & \begin{array}{l}\text { elderly } \\ \text { Pittsburgh sleep quality index }\end{array} \\ \text { PSQI } & \text { Standard deviation } \\ \text { SD } & \text { Utrecht coping list }\end{array}$

\section{Introduction}

More than 800,000 people worldwide were newly diagnosed with head and neck cancer (HNC) in 2018 [1]. There is a growing attention to maximize health-related quality of life (HRQoL) of newly diagnosed HNC patients [2]. These patients often suffer from emotional distress and concerns related to the future consequences of HNC itself and its treatment $[3,4]$, which may affect their sleep quality. Sleep quality before the start of cancer treatment is also known to be associated with HRQoL throughout the cancer trajectory $[5,6]$; thus, early detection of poor sleep quality is necessary to initiate prehabilitation strategy to optimize HRQoL during and after HNC treatment [7].

Nonetheless, little is known about sleep quality among newly diagnosed $\mathrm{HNC}$ patients. A recent systematic review found a wide prevalence range of 16 to $66 \%$ for various definitions of sleep disturbances among HNC patients before treatment [8]. Additionally, most of the included studies did not use validated instrument to measure sleep quality as reported by the patients themselves [8]. To illustrate, only two studies among newly diagnosed HNC patients used the Pittsburgh sleep quality index (PSQI) [9, 10], a validated and most widely used self-report instrument for sleep quality in clinical and non-clinical populations [11]. Using a PSQI total score cut-off of $>5$, these two studies found that $37 \%$ of their patients had poor sleep quality $[9,10]$. Since these studies only included nasopharyngeal cancer patients $[9,10]$, the prevalence of poor sleep quality among a more generalizable sample of newly diagnosed HNC patients is yet to be examined.

Furthermore, more insight is needed on the factors associated with poor sleep quality among HNC patients before treatment. Only two studies have examined this question thus far and found that age, marital status, HNC subsite, smoking status, and physical activity were significantly associated factors
$[12,13]$, implying the importance to assess these factors in sleep quality evaluation among newly diagnosed HNC patients. Neither of these studies, however, examined two important factors among newly diagnosed $\mathrm{HNC}$ patients: coping styles and HNC symptoms. Coping styles determine how someone perceives stressful life events, such as being diagnosed with cancer. Although the effectiveness of coping style may depend on the context of the stressor, certain coping styles such as avoidance coping, substance use, and behavioral and mental disengagement are found to be associated with more psychological distress among HNC patients before starting treatment [14]. Avoidance coping style in particular is found to be associated with poor sleep among cancer patients in general $[15,16]$. So far, there is no research on whether specific coping styles are associated with poor sleep quality among newly diagnosed HNC patients. Furthermore, patients recently diagnosed with $\mathrm{HNC}$ often suffer from oral pain and swallowing problems [17] which may disrupt their sleep quality.

Insight into poor sleep quality among newly diagnosed HNC patients may help healthcare providers to design a better sleep intervention for those who already need it before starting treatment. Therefore, we aimed to examine the prevalence of poor sleep quality among $\mathrm{HNC}$ patients before start of treatment and to examine the association of poor sleep quality with sociodemographic factors, clinical characteristics, lifestyle factors, coping style, and HNC-specific symptoms.

\section{Patients and methods}

\section{Study population}

Data of the prospective NETherlands QUality of life and BIomedical Cohort study in head and neck cancer (NETQUBIC) [18] was used. In the NET-QUBIC study, 739 newly diagnosed $\mathrm{HNC}$ patients from $\mathrm{HNC}$ centers in 5 university medical centers and 2 of their satellite hospitals in the Netherlands were included between March 2014 and June 2018. Inclusion criteria were as follows: being diagnosed with squamous cell carcinoma of the oral cavity, oropharynx, hypopharynx, larynx, or neck lymph node metastasis of an unknown primary tumor; being 18 years or older; having curative treatment intention; and being able to write, read, and speak Dutch. Exclusion criteria were having severe psychiatric comorbidity (e.g., schizophrenia, Korsakoff's syndrome, severe dementia), lymphoma, thyroid cancer, nasopharyngeal cancer, malignancy of skin, or malignancy of salivary glands. The NET-QUBIC study was approved by the Medical Research Ethics Committee of the coordinating center (Amsterdam UMC, location VUmc, document number: 2013.301[A2018.307]-NL45051.029.13). Detailed procedure of the NET-QUBIC study can be found elsewhere [18]. 


\section{Measures}

The ongoing NET-QUBIC study encompasses measurements at baseline and at 3-, 6-, 12-, 24-, 36-, 48-, and 60-month follow-up. In the present study, we used the baseline data, which were collected shortly after the diagnosis and before cancer treatment was started.

Sleep quality was measured using the PSQI, which was filled in by the patients themselves [19]. Its validity and reliability have been confirmed both in general population [20] and in cancer patients [21, 22]. PSQI consists of 19 items across seven components of sleep quality and disturbances: (1) subjective sleep quality (i.e., one item "How would you rate your sleep quality overall?"), (2) sleep onset latency (i.e., two items asking time needed to fall asleep and its frequency in a week; poor sleep onset is defined as needing $\geq 30 \mathrm{~min}$ to fall asleep [23]), (3) sleep duration (i.e., one item "How many hours of actual sleep do you get at night?"; $\leq 6 \mathrm{~h}$ is associated with worse survival among cancer patients [24]), (4) sleep efficiency (i.e., a percentage calculated by dividing time asleep by time spent in bed and multiplied by $100 ;<85 \%$ indicates poor efficiency [23]), (5) sleep disturbances (i.e., ten items about specific reasons for the sleep disturbances and their frequency), (6) use of sleep medication (i.e., one item "How often have you taken medicine to help you sleep?"), and (7) daytime dysfunction (i.e., two items asking the frequency of staying awake during daytime activity and the extent of difficulty to maintain enthusiasm to get things done) [19]. A total score (also called global score) is calculated by first scaling each component score into a 0 to 3 score then summing all component scores, resulting in a score ranging from 0 to 21; higher scores indicate worse sleep quality. Our main outcome, poor sleep quality, is defined by a total PSQI score of $>5[19,20]$. In addition, we examined questions in the PSQI (not included in the total score calculation) which assessed the frequency of the respondent's bed partner/ roommate noticing certain behaviors during the respondent's sleep. This information is clinically relevant as an indication of specific sleep disorders such as sleep apnea [25].

We examined the following sociodemographic factors: sex and age (from medical records), living situation (living together/alone, from interview), education level (low/middle/high according to the standard classification of education level in the Netherlands [26], from interview), and having a bed partner (yes/no, from the PSQI). The interview was conducted by trained field workers during house visit measurements. This interview also included other outcomes which were out of the scope of the present study [18]. Clinical characteristics (i.e., HNC subsite and stage, comorbidity, and performance status) were retrieved from medical records. Comorbidity was scored using the adult comorbidity evaluation (ACE-27). The ACE27 measures the number and severity of 27 medical conditions, and is summarized into four categories: no comorbidity, mild comorbidity, moderate comorbidity, or severe comorbidity [27]. The ACE-27 has been validated among HNC patients [28]. The patients' performance status (i.e., the patient's level of functioning seen from their daily activity, physical ability, and self-care) was measured using the one-item Eastern cooperative oncology group (ECOG) score, which ranges from 0 (fully active) to 4 (completely disabled) [29].

Coping styles were measured by the self-reported 47 -item Utrecht coping list (UCL) [30]. Each item ranges from 0 (never or seldom) to 3 (very often). The UCL measures active coping (i.e., evaluating the situation from all perspectives and taking action to solve the problem; 7 items), palliative reaction (i.e., finding distractions against the problem and seeking for manners to feel better; 8 items), avoidance coping (i.e., avoiding the situation and letting it to be solved by itself; 8 items), seeking social support (i.e., seeking help and understanding from the others, expressing worries; 6 items), passive coping (i.e., taking the blame for the situation, worrying about things in the past, withdrawing into oneself; 7 items), expression of emotions (i.e., expressing anger or abreaction; 3 items), and comforting thoughts (i.e., assuring one's self that things will get better, that things could have been worse, or that the others' may also have similar difficulties; 5 items) [30, 31]. For each coping style, a sum score was calculated, where a higher score indicates higher extent the specific coping style.

HNC symptoms were self-reported using the European organization for research and treatment of cancer quality of life questionnaire - HNC-specific module (EORTC QLQH\&N35) [32]. All symptom scales were included: oral pain (4 items), swallowing problems (4 items), sense problems (2 items), speech problems (3 items), trouble with social eating (4 items), trouble with social contact ( 5 items), and less sexual interest and enjoyment ( 2 items). Also, we included Likertscale single items measuring teeth problems, problems with opening mouth, dry mouth, sticky saliva, coughing, and feeling ill, as well as dichotomous single items measuring use of painkillers, use of nutritional supplements, use of feeding tube, weight loss, and weight gain. Each symptom scale and Likert-scale single item was converted into a score ranging from 0 to 100 , according to the EORTC guidelines. A higher score indicates a higher level of symptoms or problems.

The following lifestyle factors were examined: physical activity, body mass index (BMI), smoking status, and alcohol intake. Physical activity was assessed using the 13-item physical activity scale for the elderly (PASE) with total score ranging from 0 to 400; a higher score indicates higher physical activity [33]. The validity and reliability of this patientreported outcome measure has been previously confirmed among cancer patients [34]. BMI was measured by a trained field worker using a standardized procedure during house visit. Smoking status (not smoking/smoking every day at the time of assessment) and excessive alcohol consumption (> 14 units of alcohol per week for women or $>21$ units of alcohol per 
week for men [35]) were self-reported using study-specific items.

\section{Statistical analysis}

Only the participants who completed the main outcome measure (PSQI) were included in the analysis. Sociodemographic and clinical characteristics of participants who were included versus excluded from the analysis were compared using $t$ test (for continuous variables) or chi-square test (for categorical variables). For the included patients, we performed descriptive analyses: mean scores with standard deviation (SD) or medians with interquartile range (IQR) for continuous variables, and frequencies with proportions for categorical variables. We also examined the proportions and mean scores (with SD) or median (with IQR) of the PSQI total and component scores.

To examine the differences among patients with good and poor sleep, first, their sociodemographic, clinical, and lifestyle characteristics, as well as coping styles and HNC symptoms, were compared using chi-square (categorical variables) and $t$ tests or Mann-Whitney tests (continuous variables). Then, forward multivariable logistic regression analyses were performed on each category of associated factors separately (i.e., sociodemographic, coping style, clinical factors, HNC symptoms, and lifestyle factors) using a $p$ value for entry of $<0.05$. Subsequently, we performed logistic regression analyses using a forward selection of all significant variables across all categories, resulting in the final model. In all regression models, HNC symptom and item scores which ranged from 0 to 100 were rescaled into 10-point increments. All statistical analysis was performed using the IBM SPSS Statistics for Windows, Version 25.0 (Armonk, NY: IBM Corp. 2017).

\section{Results}

\section{Study population}

Among the 739 eligible patients, 179 (24\%) did not have available PSQI scores and were excluded from our analyses. Age, gender, education level, HNC subsite, and HNC stage were similar between patients with $(n=560,76 \%)$ and without PSQI data. However, patients providing PSQI data more often lived with others, had better ECOG performance status, and less comorbidity compared with the patients not providing PSQI data (Table 1). There were 171 (21\%) missing values on BMI; therefore, this variable was not included in the analysis. For all other variables, missing values ranged from $7(1.3 \%)$ to $43(7.7 \%)$. Since only 5 patients $(0.9 \%)$ used a feeding tube, we did not include this variable in the analysis.

Our final sample of patients with PSQI data were mostly men $(75 \%)$, on average 63 years old $(\mathrm{SD}=9)$, and low educated (42\%). Most patients (79\%) lived with others (i.e., with a partner, child, and/or housemate) and $70 \%$ had a bed partner. About one-third (32\%) had no comorbidity (Table 3).

\section{Sleep quality}

The median PSQI total score was $5(\mathrm{IQR}=3-8)$. There were $246 \mathrm{HNC}$ patients who were classified as having poor sleep (PSQI > 5; 44\%). The median time needed to fall asleep (i.e., sleep latency) was $13 \mathrm{~min}(\mathrm{IQR}=8-30)$ and the mean sleep duration was $7 \mathrm{~h}(\mathrm{SD}=1)$. Among all patients, $13 \%$ slept $\leq 6 \mathrm{~h}$ in a night, $42 \%$ had $<85 \%$ sleep efficiency, $16 \%$ could not fall asleep within $30 \mathrm{~min}$ at least three nights a week, and $45 \%$ reported nighttime or early morning awakening at least three times a week. At least once in a week, $15 \%$ of the patients used medication to improve their sleep and 5\% experienced difficulties staying awake during the day. Questions answered by 417 bed partners or roommates revealed that $173(43 \%)$ patients snored loudly and $13 \%$ of the patients had long breathing pause at least once a week. Mean (SD) or median (IQR), as well as the proportions of each component score, are presented in Table 2 .

\section{Factors associated with poor sleep quality}

Univariate analyses revealed that patients with poor sleep $(n=$ 246) were more often women, younger, diagnosed with cancer in the oral cavity, using painkillers, or using nutritional supplements (Table 3). Furthermore, compared with good sleepers, poor sleepers used less active coping and more palliative reaction, passive coping, and expression of emotions. Also, poor sleepers had worse scores of all HNC symptoms except for speech problems, problems with opening mouth, coughing, and weight changes.

Multivariate logistic regression models on the separate domains of risk factors showed that poor sleep was significantly associated with sociodemographic factors (younger age, being female), coping style (more passive coping), clinical characteristics (HNC subsite, especially cancer in the oral cavity and oropharynx compared with larynx), and HNC symptoms (oral pain, less sexual interest and enjoyment, and feeling ill). Combining all of these significant variables in a logistic regression model, we found that younger age (odds ratio [OR] per increasing year of age $=0.98,95 \% \mathrm{CI}=0.96-1.00, p$ value $=0.049)$, being female $(\mathrm{OR}=2.6,95 \% \mathrm{CI}=1.7-4.1, p$ value $<0.001)$, higher passive coping style $(\mathrm{OR}=1.18,95 \%$ $\mathrm{CI}=1.09-1.28, p$ value $<0.001)$, more oral pain $(\mathrm{OR}=1.10$, $95 \% \mathrm{CI}=1.01-1.19, p$ value $=0.023)$, and less sexual interest and enjoyment $(\mathrm{OR}=1.13,95 \%$ CI $1.06-1.20, p$ value $<$ $0.001)$ corresponded to greater odds of having poor sleep (Table 4). 
Table 1 Characteristics of patients with available versus unavailable PSQI data

\begin{tabular}{|c|c|c|c|}
\hline & $\begin{array}{l}\text { Available PSQI data } \\
(n=560)\end{array}$ & $\begin{array}{l}\text { Unavailable PSQI data } \\
(n=179)\end{array}$ & $p$ value \\
\hline Age (mean, SD) & $63(9)$ & $63(11)$ & 0.33 \\
\hline Women & $142(25 \%)$ & $48(27 \%)$ & 0.70 \\
\hline \multicolumn{4}{|l|}{ Education level $^{\mathrm{a}, \mathrm{b}}$} \\
\hline Low & $215(42 \%)$ & $64(49 \%)$ & \multirow[t]{3}{*}{0.19} \\
\hline Middle & $136(26 \%)$ & $35(27 \%)$ & \\
\hline High & $166(32 \%)$ & $32(24 \%)$ & \\
\hline Living alone $^{\mathrm{a}}$ & $108(21 \%)$ & $56(43 \%)$ & $<0.001$ \\
\hline \multicolumn{4}{|l|}{ HNC location } \\
\hline Oral cavity & $157(28 \%)$ & $42(24 \%)$ & \multirow[t]{5}{*}{0.34} \\
\hline Oropharyn $x^{c}$ & $198(35 \%)$ & $64(36 \%)$ & \\
\hline Hypopharynx & $35(6 \%)$ & $17(10 \%)$ & \\
\hline Larynx & $152(27 \%)$ & $53(30 \%)$ & \\
\hline Unknown primary & $18(3 \%)$ & $3(2 \%)$ & \\
\hline \multicolumn{4}{|l|}{ HNC stage } \\
\hline I & $134(24 \%)$ & $29(16 \%)$ & \multirow[t]{4}{*}{0.086} \\
\hline II & $103(18 \%)$ & $29(16 \%)$ & \\
\hline III & $90(16 \%)$ & $37(21 \%)$ & \\
\hline IV & $233(42 \%)$ & $84(47 \%)$ & \\
\hline \multicolumn{4}{|c|}{ ECOG performance status } \\
\hline 0 & $398(71 \%)$ & $109(61 \%)$ & \multirow[t]{2}{*}{0.012} \\
\hline 1 or more & $162(29 \%)$ & $70(39 \%)$ & \\
\hline \multicolumn{4}{|l|}{ Comorbidity $^{\mathrm{a}}$} \\
\hline None & $172(32 \%)$ & $32(19 \%)$ & \multirow[t]{4}{*}{0.002} \\
\hline Mild & $203(38 \%)$ & $61(37 \%)$ & \\
\hline Moderate & $109(20 \%)$ & $46(28 \%)$ & \\
\hline Severe & $50(9 \%)$ & $26(16 \%)$ & \\
\hline
\end{tabular}

${ }^{\text {a }}$ There were 91 missing values on education level, 90 missing values on living arrangement, and 40 missing values on comorbidity

${ }^{\mathrm{b}}$ Low education level includes primary education, lower or preparatory vocational education, and intermediary general secondary education. Middle education level includes senior general secondary education and higher general secondary education. High education level includes higher professional education and university

${ }^{\mathrm{c}}$ Human papilloma virus (HPV) status of oropharynx cancer patients with available PSQI data was positive among 104 patients, negative among 67 patients, and not tested among 27 patients. For patients with unavailable PSQI data, the HPV status was positive among 26 patients, negative among 32 patients, and not tested among 6 patients

\section{Discussion}

In this study, we examined the prevalence of poor sleep quality among $\mathrm{HNC}$ patients before start of treatment and investigated the associated factors. Almost half of the patients in this study were categorized as poor sleepers. This prevalence is higher than prevalence in the general population (36\%) [20], thus emphasizing the importance to incorporate sleep evaluation shortly after HNC diagnosis. Regarding the PSQI component scores, we found that a high proportion of patients reported poor sleep efficiency, difficulty to fall asleep, and nighttime or early morning awakenings; these three complaints are particularly relevant as they are the most commonly reported symptoms of insomnia [23]. Comparing our prevalence rates with those of similar studies was not possible because no other study has been published using both a similar population of HNC patients and a validated self-reported measure for sleep quality.

We found that younger age, being female, having passive coping style, more oral pain, and less sexual interest and enjoyment were the most significant factors associated with poor sleep. In the general population, older adults tend to experience age-related changes in sleep-wake architecture, such as more sleep awakenings, less deep sleep, and less sleep efficiency [23]. This is apparently not the case for HNC patients, as we found that it was younger patients who had worse sleep 
Table 2 Overview of PSQI components

PSQI component

Subjective sleep quality ${ }^{\mathrm{a}}$

Component score

Sleep latency, in minutes ${ }^{b}$

Component score*

0 (very good)

1 (fairly good)

2 (fairly bad)

3 (very bad)

0

1

2

3

Sleep duration, in hours ${ }^{\mathrm{c}}$

Component score

Sleep efficiency ${ }^{\mathrm{d}}$

Component score*

Sleep disturbances ${ }^{\mathrm{e}}$

Component score*

0 (> $7 \mathrm{~h})$

$1(6-7 \mathrm{~h})$

$2(5-6 \mathrm{~h})$

3 (<5 h)

$0(>85 \%)$

$1(75-84 \%)$

$2(65-74 \%)$

$3(<65 \%)$

0

1

2

3

Use of sleep medication $^{\mathrm{a}}$

Component score

Daytime dysfunction $^{\mathrm{a}}$

Component score*

0 (not during the past month)

1 (<1/week)

2 (1-2/week)

3 ( $\geq 3 /$ week)

Mean (SD) or median (IQR)

NA

137 (25)

303 (54)

107 (19)

12 (2)

$13(8-30)$

208 (39)

196 (36)

87 (16)

47 (9)

$7.1(1.3)$

365 (65)

122 (22)

44 (8)

$26(5)$

NA

322 (58)

125 (23)

42 (7)

64 (12)

NA

23 (4)

328 (59)

185 (33)

22 (4)

NA

460 (82)

15 (3)

28 (5)

56 (10)

NA

256 (46)

50 (9)

$3(0.5)$

NA

4 (1)

*Component score is calculated from $\geq 2$ items and rescaled to 0 to 3 . Higher component score denotes worse complaints

${ }^{\text {a }}$ Subjective sleep quality, use of sleep medication, and day dysfunction due to sleepiness could not be calculated for 1 patient

${ }^{\mathrm{b}}$ Sleep latency could not be calculated for 22 patients

${ }^{\mathrm{c}}$ Sleep duration could not be calculated for 3 patients

${ }^{\mathrm{d}}$ Sleep efficiency could not be calculated for 7 patients

${ }^{\text {e }}$ Sleep disturbances could not be calculated for 2 patients

${ }^{\mathrm{f}}$ Missing from 11 patients on snoring, 16 on breathing pauses, 14 on legs twitching, and 12 on episodes of disorientation

Abbreviations: PSQI, Pittsburgh Sleep Quality Index; NA, not applicable; SD, standard deviation 
Table 3 Characteristics and group comparisons of patients with good sleep versus patients with poor sleep

\begin{tabular}{|c|c|c|c|c|}
\hline & All patients $(n=560)$ & Good sleep $(n=314)$ & Poor sleep $(n=246)$ & $p$ value \\
\hline Age (mean, SD) & $63(9)$ & $64(9)$ & $62(9)$ & 0.020 \\
\hline Women, $n(\%)$ & $142(25)$ & $51(36)$ & $91(64)$ & 0.001 \\
\hline \multicolumn{5}{|l|}{ Education, $n(\%)^{\mathrm{a}, \mathrm{b}}$} \\
\hline Low & $215(42)$ & $117(54)$ & $98(46)$ & \multirow[t]{3}{*}{0.40} \\
\hline Middle & $136(26)$ & $84(62)$ & $52(38)$ & \\
\hline High & $166(32)$ & $95(57)$ & $71(43)$ & \\
\hline Living alone, $n(\%)^{\mathrm{a}}$ & $108(21)$ & $59(55)$ & $49(45)$ & 0.59 \\
\hline Had bed partner, $n(\%)^{\mathrm{a}}$ & $385(70)$ & $223(58)$ & $162(42)$ & 0.16 \\
\hline \multicolumn{5}{|l|}{ Coping, mean (SD) or median (IQR) ${ }^{\mathrm{a}}$} \\
\hline Active coping & $11.7(4)$ & $12(4)$ & $11.3(4)$ & 0.035 \\
\hline Palliative reaction & $9.3(3)$ & $9(4)$ & $9.8(4)$ & 0.006 \\
\hline Avoidance coping & $7.3(4)$ & $7(3)$ & $7.5(3)$ & 0.068 \\
\hline Seeking social support & $6.9(3)$ & $6.8(3)$ & $7.1(3)$ & 0.27 \\
\hline Passive coping & $3(1-5)$ & $2(1-4)$ & $3(2-6)$ & $<0.001$ \\
\hline Expression of emotions & $2(1-3)$ & $1(0-3)$ & $2(1-3)$ & 0.001 \\
\hline Comforting thoughts & $7.3(2)$ & $7.1(2)$ & $7.5(3)$ & 0.078 \\
\hline \multicolumn{5}{|l|}{ HNC location, $n(\%)$} \\
\hline Oral cavity & $157(28)$ & $74(47)$ & $83(53)$ & \multirow[t]{5}{*}{0.008} \\
\hline Oropharynx & $198(35)$ & $108(55)$ & $90(45)$ & \\
\hline Hypopharynx & $35(6)$ & $19(54)$ & $16(46)$ & \\
\hline Larynx & $152(27)$ & $103(68)$ & $49(32)$ & \\
\hline Unknown primary & $18(3)$ & $10(56)$ & $8(44)$ & \\
\hline \multicolumn{5}{|l|}{ HNC stage, $n(\%)$} \\
\hline I & $134(24)$ & $84(63)$ & $50(37)$ & \multirow[t]{4}{*}{0.24} \\
\hline II & $103(18)$ & $59(57)$ & $44(43)$ & \\
\hline III & $90(16)$ & $45(50)$ & $45(50)$ & \\
\hline IV & $233(42)$ & $126(54)$ & $107(46)$ & \\
\hline \multicolumn{5}{|l|}{ ECOG performance status, $n(\%)$} \\
\hline 0 & $398(71)$ & $231(58)$ & $167(42)$ & \multirow[t]{2}{*}{0.16} \\
\hline 1 or more & $162(29)$ & $83(51)$ & $79(49)$ & \\
\hline \multicolumn{5}{|l|}{ Comorbidity, $n(\%)^{\mathrm{a}}$} \\
\hline None & $172(32)$ & $102(59)$ & $70(41)$ & \multirow[t]{4}{*}{0.42} \\
\hline Mild & $203(38)$ & $113(56)$ & $90(44)$ & \\
\hline Moderate & $109(20)$ & $62(57)$ & $47(43)$ & \\
\hline Severe & $50(9)$ & $23(46)$ & $27(54)$ & \\
\hline \multicolumn{5}{|c|}{ Head-neck symptoms, mean (SD) or $n(\%)^{\mathrm{a}}$} \\
\hline Oral pain & $26(24)$ & $22(22)$ & $32(26)$ & $<0.001$ \\
\hline Swallowing problems & $16(21)$ & $13(20)$ & $19(22)$ & $<0.001$ \\
\hline Sense problems & $8(17)$ & $7(14)$ & $10(19)$ & 0.008 \\
\hline Speech problems & $19(23)$ & $17(22)$ & $20(24)$ & 0.16 \\
\hline Problems with social eating & $11(17)$ & $8(15)$ & $15(19)$ & $<0.001$ \\
\hline Problems with social contact & $4(10)$ & $3(7)$ & $6(12)$ & $<0.001$ \\
\hline Less sexual interest and enjoyment & $26(31)$ & $20(27)$ & $34(34)$ & $<0.001$ \\
\hline Teeth problems & $16(27)$ & $13(25)$ & $19(30)$ & 0.013 \\
\hline Problems with opening mouth & $12(25)$ & $10(22)$ & $14(28)$ & 0.053 \\
\hline Dry mouth & $16(23)$ & $13(21)$ & $19(25)$ & 0.001 \\
\hline Sticky saliva & $14(24)$ & $12(22)$ & $17(25)$ & 0.017 \\
\hline Coughing & $23(24)$ & $22(22)$ & $25(26)$ & 0.11 \\
\hline Feeling ill & $13(23)$ & $9(18)$ & $18(24)$ & $<0.001$ \\
\hline
\end{tabular}


Table 3 (continued)

\begin{tabular}{|c|c|c|c|c|}
\hline & All patients $(n=560)$ & Good sleep $(n=314)$ & Poor sleep $(n=246)$ & $p$ value \\
\hline Used painkillers $^{\mathrm{c}}$ & $299(54 \%)$ & $141(47 \%)$ & $158(53 \%)$ & $<0.001$ \\
\hline Used nutritional supplements $^{\mathrm{c}}$ & $86(16 \%)$ & $36(42 \%)$ & $50(58 \%)$ & 0.006 \\
\hline Had weight loss ${ }^{\mathrm{c}}$ & $133(24 \%)$ & $65(49 \%)$ & $68(51 \%)$ & 0.056 \\
\hline Had weight gain ${ }^{\mathrm{c}}$ & $57(10 \%)$ & $29(51 \%)$ & $28(49 \%)$ & 0.40 \\
\hline Smoking daily, $n(\%)^{\mathrm{a}}$ & $123(22)$ & $62(50)$ & $61(50)$ & 0.18 \\
\hline Excessive alcohol consumption, $n(\%)^{\mathrm{a}}$ & $124(22)$ & $73(59)$ & $51(41)$ & 0.54 \\
\hline PASE global score, median (IQR) ${ }^{\mathrm{a}}$ & $84(43-144)$ & $83(46-134)$ & $90(39-154)$ & 0.51 \\
\hline
\end{tabular}

${ }^{a}$ Variables with missing values: 43 missing values on education, 42 on living arrangements, 7 on having bed partner, 4-10 on each UCL domain scores, 26 on comorbidity, 7 on all EORTC-H\&N35 domains (except less sexual interest and enjoyment [missing $=43$ ], teeth problems [missing $=12$ ], problems with opening mouth [missing =9], sticky saliva [missing $=8$ ], coughing [missing = 8], using painkillers [missing = 10], using feeding tube [missing = 8], had weight loss [missing = 11], and had weight gain [missing = 14]), 9 on smoking status, 7 on alcohol consumption, 7 on PASE score, 11 on snoring, and 16 on breathing pauses

${ }^{\mathrm{b}}$ Low education level includes primary education, lower or preparatory vocational education, and intermediary general secondary education. Middle education level includes senior general secondary education and higher general secondary education. High education level includes higher professional education and university

c Item with yes/no answer response; the proportions of "yes" were reported in the table

Abbreviations: $H N C$, head and neck cancer; PASE, physical activity scale for the elderly; $S D$, standard deviation; $U C L$, Utrecht Coping List

quality. This is in line with findings from two previous studies among HNC patients before treatment $[12,13]$. These findings may be attributed to fear of cancer progression which is higher among younger HNC patients before treatment [36]. Patients who are diagnosed with cancer at a younger age also experience higher psychological distress compared with their older counterparts [37], which may contribute to their higher risk of having poor sleep.

The greater odds of having poor sleep among women in our study is consistent with findings among general population in the Netherlands [38]. Differences in the physiology of sex hormones as well as circadian rhythms among men and women may explain the differences of sleep quality between

Table 4 Final model of the multivariable logistic regression analyses with poor sleep (PSQI total score $>5)$ as outcome $(n=516)$

\begin{tabular}{llrc}
\hline Covariates $^{\mathrm{a}}$ & OR & $p$ value & \multicolumn{1}{c}{$95 \%$ CI } \\
\hline Age & 0.98 & 0.049 & $0.96-1.00$ \\
Female & 2.6 & $<0.001$ & $1.7-4.1$ \\
Passive coping & 1.18 & $<0.001$ & $1.09-1.28$ \\
Oral pain $^{\mathrm{b}}$ & 1.10 & 0.023 & $1.01-1.19$ \\
Less sexual interest and enjoyment $^{\mathrm{b}}$ & 1.13 & $<0.001$ & $1.06-1.20$ \\
\hline
\end{tabular}

${ }^{\text {a }}$ Covariates retained in the final model is based on forward selection of all significant variables of each category, with entry criteria $p$ value of $<$ 0.05 . Nagelkerke's $R=0.21$

${ }^{\mathrm{b}}$ Oral pain and less sexual interest and enjoyment scores were transformed into 10-point increments

Abbreviations: CI, confidence interval; OR, odds ratio; PSQI, Pittsburgh Sleep Quality Index the sexes [39]. However, previous studies examining poor sleep among pre-treated HNC patients reported different findings. The cause of this disagreement is unclear, although it may be in part due to the differences in defining sleep quality and the associated factors examined in the study. Using a single item ("How much is a problem is sleeping for you?"), Zhou and colleagues compared sex proportions of patients with severe sleep problem $(n=45)$ with those who had no, mild, or moderate problems ( $n=281)$; they found that slightly more females had severe sleep problems, although this was not statistically significant [13]. These findings suggest that a dichotomized Likert-scale item is not informative enough to illustrate sex differences in poor sleep quality. On the other hand, Duffy and colleagues, who also looked at depressive symptoms in relation to sleep quality, found that depressive symptoms, and not sex, are associated with sleep quality [12]. Whether sex differences on sleep quality are completely attributed to depressive symptoms remains questionable, since depressive symptoms among both men and women seem to affect sleep quality in different ways [40, 41]. More research is needed to assess the complex relationship of depressive symptoms and sleep quality among men and women with HNC.

We found a significant association between poor sleep and passive coping style. Diagnosis of cancer, as a form of distress, may be perceived differently among HNC patients and its effect on sleep quality may be determined by coping style. No specific coping style is inherently positive or negative since certain coping styles can be effective respective of the onset and context of the distress [42]. However, coping strategies such as avoidance, denial, substance use, abreaction, and behavioral and mental disengagement are known to be related 
to psychological distress among newly diagnosed HNC patients $[14,43]$. We found that sleep quality before HNC treatment is associated with passive coping style, which comprises worrying about things in the past, withdrawing into oneself, and taking the blame for the situation. Passive coping style is also found to be associated with psychological distress after HNC treatment [44]. Although our cross-sectional study may not be able to explain any causal relationship, a longitudinal research among general population found that substance use, behavioral disengagement, and distracting oneself, compared with other coping styles, are associated with higher risk of having insomnia after stress exposure [45]. Whether this is also the case for HNC patients after treatment starts remains to be examined in future research.

As expected, based on clinical practice, we found significant associations between poor sleep and HNC symptoms, more specifically oral pain and less sexual enjoyment. The relationship between pain, sexual problems, and sleep problems may be bidirectional. First of all, pain may disrupt sleep, while poor sleep may also lower one's pain threshold, thus increasing the perception of pain [46]. Sexual satisfaction may improve sleep quality through the release of oxytocin and prolactin levels, both of which are neuropeptides involved in sleep regulation [47]. Lack of sleep, on the other hand, may result in fatigue and reduce sexual desire [48]. More research is needed to confirm whether sleep intervention before treatment can optimize symptom management in HNC patients throughout the cancer trajectory.

\section{Strength and limitations}

The strength of this study was that we used a large sample of $560 \mathrm{HNC}$ patients from multiple hospitals in the Netherlands to investigate the association between sleep problems and various sociodemographic, clinical, and lifestyle factors as well as coping style and HNC symptoms. Moreover, our study was the first study so far to report on the prevalence of different components of sleep quality (as measured by the PSQI) among HNC patients before starting treatment. However, we found differences between patients who were included because their PSQI data were available $(n=560)$ compared with those that did not $(n=179)$; our study population consists of HNC patients with better performance status and less severe comorbidity. Our study may, therefore, underestimate the real prevalence of poor sleep quality among newly diagnosed HNC patients. In addition, the NET-QUBIC participants were not completely representative for the overall HNC population in the Netherlands [18], which may limit generalizability of our study findings to all HNC patients.

\section{Conclusions}

Poor sleep quality is highly prevalent among newly diagnosed $\mathrm{HNC}$ patients and is associated with younger age, being female, passive coping style, more oral pain, and less sexual interest and enjoyment. Our findings underline the need for early sleep evaluation among HNC patients already before starting treatment, as well as taking coping styles and HNC symptoms into consideration when implementing sleep intervention.

Funding information This study was carried out using the research infrastructure within the NETherlands QUality of life and BIomedical Cohort study in head and neck cancer (NET-QUBIC) project funded by the Dutch Cancer Society (grant number VU 2013-5930). The funding body had no role in the study design, the data collection, analysis, and interpretation, nor the manuscript preparation.

Compliance with ethical standards The NET-QUBIC study was approved by the Medical Research Ethics Committee of the coordinating center (Amsterdam UMC, location VUmc, document number: 2013.301[A2018.307]-NL45051.029.13). Participants provided written informed consent to use and re-use their data and samples in future studies that aim to research HRQOL and improved diagnosis and treatment of HNC. The NET-QUBIC Research Agreement is signed by all participating hospitals.

Conflict of interest The authors declare that they have no conflict of interest.

Open Access This article is licensed under a Creative Commons Attribution 4.0 International License, which permits use, sharing, adaptation, distribution and reproduction in any medium or format, as long as you give appropriate credit to the original author(s) and the source, provide a link to the Creative Commons licence, and indicate if changes were made. The images or other third party material in this article are included in the article's Creative Commons licence, unless indicated otherwise in a credit line to the material. If material is not included in the article's Creative Commons licence and your intended use is not permitted by statutory regulation or exceeds the permitted use, you will need to obtain permission directly from the copyright holder. To view a copy of this licence, visit http://creativecommons.org/licenses/by/4.0/.

\section{References}

1. Bray F, Ferlay J, Soerjomataram I, Siegel RL, Torre LA, Jemal A (2018) Global cancer statistics 2018: GLOBOCAN estimates of incidence and mortality worldwide for 36 cancers in 185 countries. CA Cancer J Clin 68(6):394-424. https://doi.org/10.3322/caac. 21492

2. Smith JD, Shuman AG, Riba MB (2017) Psychosocial issues in patients with head and neck cancer: an updated review with a focus on clinical interventions. Curr Psychiatry Rep 19(9):56

3. Fingeret MC, Vidrine DJ, Reece GP, Gillenwater AM, Gritz ER (2010) Multidimensional analysis of body image concerns among newly diagnosed patients with oral cavity cancer. Head Neck 32(3): 301-309

4. Posluszny DM, Dougall AL, Johnson JT, Argiris A, Ferris RL, Baum A, Bovbjerg DH, Dew MA (2015) Posttraumatic stress 
disorder symptoms in newly diagnosed patients with head and neck cancer and their partners. Head Neck 37(9):1282-1289

5. Clevenger L, Schrepf A, Degeest K, Bender D, Goodheart M, Ahmed A, Dahmoush L, Penedo F, Lucci J 3rd, Thaker PH, Mendez L, Sood AK, Slavich GM, Lutgendorf SK (2013) Sleep disturbance, distress, and quality of life in ovarian cancer patients during the first year after diagnosis. Cancer 119(17):3234-3241. https://doi.org/10.1002/cncr.28188

6. Dean GE, Sabbah EA, Yingrengreung S, Ziegler P, Chen H, Steinbrenner LM, Dickerson SS (2015) Sleeping with the enemy: sleep and quality of life in patients with lung cancer. Cancer Nurs 38(1):60-70

7. Chen D, Yin Z, Fang B (2018) Measurements and status of sleep quality in patients with cancers. Support Care Cancer 26(2):405414. https://doi.org/10.1007/s00520-017-3927-x

8. Santoso AM, Jansen F, de Vries R, Leemans CR, van Straten A, Verdonck-de Leeuw IM (2019) Prevalence of sleep disturbances among head and neck cancer patients: a systematic review and meta-analysis. Sleep Med Rev 47:62-73

9. Mo YL, Li L, Qin L, Zhu XD, Qu S, Liang X, Wei ZJ (2014) Cognitive function, mood, and sleep quality in patients treated with intensity-modulated radiation therapy for nasopharyngeal cancer: a prospective study. Psycho-Oncology 23(10):1185-1191

10. Qin L, Mo Y-L, Li L, Wei Z-J, Zhu X-D, Yin X, Wei Q (2015) Sleep characteristics and psychological symptoms in patients with locally advanced nasopharyngeal carcinoma before and after intensity-modulated radiotherapy and concurrent chemotherapy. Psychol Health Med 20(6):662-669

11. Mollayeva T, Thurairajah P, Burton K, Mollayeva S, Shapiro CM, Colantonio A (2016) The Pittsburgh sleep quality index as a screening tool for sleep dysfunction in clinical and non-clinical samples: a systematic review and meta-analysis. Sleep Med Rev 25:52-73. https://doi.org/10.1016/j.smrv.2015.01.009

12. Duffy SA, Khan MJ, Ronis DL, Fowler KE, Gruber SB, Wolf GT, Terrell JE (2008) Health behaviors of head and neck cancer patients the first year after diagnosis. Head Neck 30(1):93-102

13. Zhou ES, Clark K, Recklitis CJ, Obenchain R, Loscalzo M (2018) Sleepless from the get go: sleep problems prior to initiating cancer treatment. Int J Behav Med 25(5):502-516. https://doi.org/10.1007/ s12529-018-9715-2

14. Morris N, Moghaddam N, Tickle A, Biswas S (2018) The relationship between coping style and psychological distress in people with head and neck cancer: a systematic review. Psycho-Oncology 27(3):734-747. https://doi.org/10.1002/pon.4509

15. Hoyt MA, Thomas KS, Epstein DR, Dirksen SR (2009) Coping style and sleep quality in men with cancer. Ann Behav Med 37(1): 88-93

16. Thomas KS, Bower J, Hoyt MA, Sepah S (2010) Disrupted sleep in breast and prostate cancer patients undergoing radiation therapy: the role of coping processes. Psychooncology 19(7):767-776. https://doi.org/10.1002/pon.1639

17. Molassiotis A, Rogers M (2012) Symptom experience and regaining normality in the first year following a diagnosis of head and neck cancer: a qualitative longitudinal study. Palliat Support Care 10(3):197-204

18. Verdonck-de Leeuw IM, Jansen F, Brakenhoff RH, Langendijk JA, Takes R, Terhaard CHJ, de Jong RJB, Smit JH, Leemans CR (2019) Advancing interdisciplinary research in head and neck cancer through a multicenter longitudinal prospective cohort study: the NETherlands QUality of life and BIomedical Cohort (NETQUBIC) data warehouse and biobank. BMC Cancer 19(1):765. https://doi.org/10.1186/s12885-019-5866-Z

19. Buysse DJ, Reynolds CF 3rd, Monk TH, Berman SR, Kupfer DJ (1989) The Pittsburgh Sleep Quality Index: a new instrument for psychiatric practice and research. Psychiatry Res 28(2):193-213
20. Hinz A, Glaesmer H, Brähler E, Löffler M, Engel C, Enzenbach C, Hegerl U, Sander C (2017) Sleep quality in the general population: psychometric properties of the Pittsburgh Sleep Quality Index, derived from a German community sample of 9284 people. Sleep Med 30:57-63. https://doi.org/10.1016/j.sleep.2016.03.008

21. Beck SL, Schwartz AL, Towsley G, Dudley W, Barsevick A (2004) Psychometric evaluation of the Pittsburgh Sleep Quality Index in cancer patients. J Pain Symptom Manag 27(2):140-148

22. Tzeng JI, Fu Y-W, Lin C-C (2012) Validity and reliability of the Taiwanese version of the Pittsburgh Sleep Quality Index in cancer patients. Int J Nurs Stud 49(1):102-108. https://doi.org/10.1016/j. ijnurstu.2011.08.004

23. Morin CM, Espie CA (2007) Clinical features of insomnia. In: Insomnia: a clinical guide to assessment and treatment Springer Science \& Business Media, p 16

24. Collins KP, Geller DA, Antoni M, Donnell DM, Tsung A, Marsh JW, Burke L, Penedo F, Terhorst L, Kamarck TW, Greene A, Buysse DJ, Steel JL (2017) Sleep duration is associated with survival in advanced cancer patients. Sleep Med 32:208-212. https:// doi.org/10.1016/j.sleep.2016.06.041

25. Fedson AC, Pack AI, Gislason T (2012) Frequently used sleep questionnaires in epidemiological and genetic research for obstructive sleep apnea: a review. Sleep Med Rev 16(6):529-537

26. (CBS) SN (2016) Standaard Onderwijsindeling 2016. CBS Statline. https://www.cbs.nl/nl-nl/onze-diensten/methoden/classificaties/ onderwijs-en-beroepen/standaard-onderwijsindeling $\% 2 \mathrm{D} \%$ 2Dsoi\%2D\%2D/standaard-onderwijsindeling-2016.

27. Piccirillo J, Costas I, Claybour P, Borah A, Grove L, Jeffe D (2003) The measurement of comorbidity by cancer registries. J Registry Manag 30(1):8-15

28. Piccirillo JF (2000) Importance of comorbidity in head and neck cancer. Laryngoscope 110(4):593-602. https://doi.org/10.1097/ 00005537-200004000-00011

29. Oken MM, Creech RH, Tormey DC, Horton J, Davis TE, McFadden ET, Carbone PP (1982) Toxicity and response criteria of the Eastern Cooperative Oncology Group. Am J Clin Oncol 5(6):649-655

30. Schreurs P, Van de Willige G, Brosschot J, Tellegen B, Graus G (1993) De Utrechtse Coping Lijst (Utrecht Coping Questionnaire). Lisse: Swets and Zeitlinger,

31. Hoekstra-Weebers JE, Wijnberg-Williams BJ, Jaspers JP, Kamps WA, van de Wiel HB (2012) Coping and its effect on psychological distress of parents of pediatric cancer patients: a longitudinal prospective study. Psycho-Oncology 21(8):903-911

32. Bjordal K, Ahlner-Elmqvist M, Tollesson E, Jensen AB, Razavi D, Maher EJ, Kaasa S (1994) Development of a European Organization for Research and Treatment of Cancer (EORTC) questionnaire module to be used in quality of life assessments in head and neck cancer patients. Acta Oncol 33(8):879-885

33. Washburn RA, Smith KW, Jette AM, Janney CA (1993) The Physical Activity Scale for the Elderly (PASE): development and evaluation. J Clin Epidemiol 46(2):153-162

34. Liu RD, Buffart LM, Kersten MJ, Spiering M, Brug J, van Mechelen W, Chinapaw MJ (2011) Psychometric properties of two physical activity questionnaires, the AQuAA and the PASE, in cancer patients. BMC Med Res Methodol 11(1):30

35. (CBS) SN (2019) Lifestyle and (preventive) health examination; personal characteristics. CBS Statline. https://opendata.cbs.nl/ statline/\#/CBS/en/dataset/83021ENG/table?ts=1580381653038. Accessed 13 August 2019

36. Miroševič Š, Prins JB, Selič P, Zaletel Kragelj L, Klemenc Ketiš Z (2019) Prevalence and factors associated with unmet needs in posttreatment cancer survivors: a systematic review. Eur J Cancer Care 28(3):e13060. https://doi.org/10.1111/ecc.13060 
37. Krok JL, Baker TA, McMillan SC (2013) Age differences in the presence of pain and psychological distress in younger and older cancer patients. J Hosp Palliat Nurs 15(2):107-113

38. Kerkhof GA (2017) Epidemiology of sleep and sleep disorders in the Netherlands. Sleep Med 30:229-239

39. Mong JA, Cusmano DM (2016) Sex differences in sleep: impact of biological sex and sex steroids. Philos Trans R Soc B Biol Sci 371(1688):20150110

40. Khan AA, Gardner CO, Prescott CA, Kendler KS (2002) Gender differences in the symptoms of major depression in opposite-sex dizygotic twin pairs. Am J Psychiatr 159(8):1427-1429

41. Montagni I, Qchiqach S, Pereira E, Tully PJ, Tzourio C (2018) Sexspecific associations between sleep and mental health in university students: a large cross-sectional study. J Am Coll Heal 68:1-8

42. Lazarus RS (1993) Coping theory and research: past, present, and future. Fifty years of the research and theory of RS Lazarus: An analysis of historical and perennial issues:366-388

43. Sherman AC, Simonton S, Adams DC, Vural E, Hanna E (2000) Coping with head and neck cancer during different phases of treatment. Head Neck 22(8):787-793

44. Verdonck-de Leeuw IM, Eerenstein SE, Van der Linden MH, Kuik DJ, De Bree R, Leemans CR (2007) Distress in spouses and patients after treatment for head and neck cancer. Laryngoscope 117(2): 238-241

45. Pillai V, Roth T, Mullins HM, Drake CL (2014) Moderators and mediators of the relationship between stress and insomnia: stressor chronicity, cognitive intrusion, and coping. Sleep 37(7):11991208A. https://doi.org/10.5665/sleep.3838

46. Wei Y, Blanken TF, Van Someren EJW (2018) Insomnia really hurts: effect of a bad night's sleep on pain increases with insomnia severity. Front Psych 9(377). https://doi.org/10.3389/fpsyt.2018. 00377

47. Lastella M, O’Mullan C, Paterson JL, Reynolds AC (2019) Sex and sleep: perceptions of sex as a sleep promoting behavior in the general adult population. Front Public Health 7(33). https://doi.org/10. 3389/fpubh.2019.00033

48. Holloway V, Wylie K (2015) Sex drive and sexual desire. Curr Opin Psychiatry 28(6):424-429. https://doi.org/10.1097/yco. 0000000000000199

Publisher's note Springer Nature remains neutral with regard to jurisdictional claims in published maps and institutional affiliations. 